\title{
Is need enough?
}

\author{
If a marketing \\ approach were truly \\ embraced by the \\ profession we might \\ find that instead of \\ having too many \\ dentists, in the future \\ we will have too few.
}

\section{This year, on 17 April, the Jean Todd lecture at the Dental Practice Board Conference $\mathbf{2 0 0 0}$ was given by Professor Aubrey Sheiham. It was provocatively titled 'Do we need so many dentists?' and has attracted a good deal of atten- tion, partly because one of his suggestions is that we will need fewer dentists (and hygienists) in the future if the current trends of reducing dental disease continue.}

While we have all been taught at dental school that our ultimate aim is to reduce dental disease in the population (having first treated the damage caused) the idea that a reduction in dental manpower automatically follows a reduction in dental needs in the community is not necessarily correct. This concept is based on the traditional approach to patient care that we have followed for many years. We assume that patient need is more important than what patients want, and worse we assume that the needs that we identify are the really important criteria for the provision of patient care.

Focusing on want rather than need is not just a play on words - it is a different philosophy. Identifying the wants of a group of people and then satisfying them is what marketing is all about. Sadly professions have often believed that marketing is about persuading people to buy (even when they do not need what is on offer). In my opinion marketing can be said to be more ethical than traditional healthcare models because the former actually finds out what people want while the latter is based simply on need identified by the dentist.

The usual argument against a marketing approach is that patients often do not know what they want or may want inappropriate treatment (such as extractions that are clinically not needed). The key to ensuring that a marketing approach is not misused by the patient is to focus on outcome rather than process. The role of the professional is to provide the appropriate process to achieve the outcome the patient wants, in other words the dentist uses his or her clinical expertise in diagnosis and treatment. The important thing to realise is that what dentists want to provide because there is a dental need, and what dentists would actually provide if they focussed on what patients want is usually the same dentistry in both cases.

The point here is that the dental profession has been less effective than it could have been at really identifying what people want from dentistry, both on an individual level and a community level. I believe there is far more potential for the provision of dental care for many more people than happens at the moment because we have not followed marketing principles and we have been very poor at selling what we can offer. If a marketing approach were truly embraced by the profession we might find that instead of having too many dentists, in the future we will have too few. If you add to this the fact that the principles of basing dental care on need rather than want is actually less ethical an approach then it begs the question - should we continue to follow the traditions of the past? They are less effective and less ethical than a marketing approach that puts the patient's requirements where they should be - with the patient.

Professor Sheiham has delivered a lecture that has set dentistry talking. It is time to do more than just talk; we must do some serious thinking because the future of the profession and the care we offer to patients are both involved.

The final choice is ours to make. We must decide whether, as a profession, we wish to continue with our approach to dental care based on need and the implications to ourselves and our patients. The alternative is to focus our thoughts on dental care based on want that is truly market-led, which I believe will create new and rewarding potential for both patients and the dental team. Which is it to be? That is the real question for us to ponder. 